\title{
Liquen escleroso genital y extragenital. Reporte de un caso con buena respuesta a la fototerapia ultravioleta $B$ de banda angosta
}

\section{Genital and extragenital lichen sclerosus. Report of a case succesfully treated with narrowband UV-B phototherapy}

\author{
Matias E. Williner ${ }^{1 *}$, Manuela Inza ${ }^{1}$, Iliana S. Garay ${ }^{1}$, Maria Kurpis ${ }^{2}$ y Alejandro Ruiz-Lascano ${ }^{1}$
}

${ }^{1}$ Servicio de Dermatología; ${ }^{2}$ Servicio de Anatomía Patológica. Hospital Privado Universitario de Córdoba, Córdoba, Argentina

\section{Resumen}

El liquen escleroso es una enfermedad inflamatoria crónica, de etiología desconocida, que afecta la piel y las mucosas. Presentamos un caso de una paciente con liquen escleroso genital y extragenital concomitante, que presentó buena respuesta al tratamiento con clobetasol y tracrolimus tópico en la región genital, y fototerapia ultravioleta $B$ de banda angosta (UVB-BA) en las lesiones extragenitales.

Palabras clave: Liquen escleroso. Extragenital. Fototerapia.

\begin{abstract}
Lichen sclerosus is a chronic inflammatory disease of unknown etiology that affects the skin and mucous membranes. We report a case of a patient with concomitant genital and extragenital lichen sclerosus, who was successfully treated with topical clobetasol and tacrolimus in the genital area and had a good response with narrowband ultraviolet B phototherapy in the extragenital lesions.
\end{abstract}

Key words: Lichen sclerosus. Extragenital. Phototherapy.

\section{Introducción}

El liquen escleroso (LE) es un trastorno inflamatorio crónico, raro, que puede comprometer tanto el área genital como extragenital ${ }^{1,2}$.

Se presenta con mayor frecuencia en la mujer que en el hombre, con una relación de seis a uno ${ }^{3}$.

El tratamiento de primera línea actual son los corticosteroides tópicos de alta potencia. En casos resistentes, se puede recurrir a retinoides orales, metotrexato y fototerapia ${ }^{4}$.

\section{Presentación del caso}

Paciente de 88 años, sexo femenino, jubilada, con antecedente de hipertensión arterial y trastorno de ansiedad.

Consulta en el servicio de dermatología por prurito vulvar de 6 meses de evolución.

En el examen físico se constata en la región vulvar ulceración de bordes indurados de aproximadamente $2.5 \mathrm{~cm}$ de diámetro, acompañada de eritema perilesional, y palidez blanquecina que se extiende a la región perineal y perianal (Fig. 1). 
Además, en ambos flancos presenta placas atróficas, hipopigmentadas, con coloración eritematoviolácea perilesional (Fig. 2).

Los diagnósticos diferenciales planteados fueron: liquen escleroso genital y extragenital, carcinoma espinocelular en la vulva, micosis fungoide, morfea y atrofodermia.

Se decide realizar biopsia de las lesiones en la vulva y los flancos. En el examen histopatológico se observa la epidermis adelgazada con focos de vacuolización de la capa basal, acompañada en la dermis subyacente de un infiltrado inflamatorio de tipo linfocitario y homogeneización del colágeno dérmico superficial (Figs. 3 y 4).

Con los hallazgos histológicos se confirma el diagnóstico de liquen escleroso genital y extragenital.

Se solicita análisis de laboratorio con hemograma, función hepática, renal y tiroidea, que arroja resultados normales.

Se indica tratamiento tópico con clobetasol ungüento al $0.05 \%$ una vez al día durante 2 meses. Luego, en el mes siguiente, se agrega tacrolimus ungüento al $0.1 \%$ una vez al día alternado con clobetasol. En su evolución presenta disminución del prurito vulvar con resolución de la úlcera (Fig. 5). Después de 6 meses, las placas de los flancos mejoran el aspecto, pero muestran extensión del eritema y aparecen lesiones nuevas con las mismas características en la región escapular bilateral y en la raíz de los muslos.

Debido a la extensión de las lesiones extragenitales se rota su tratamiento a fototerapia ultravioleta $B$ de banda angosta (UVB-BA), mientras continúa con el tratamiento tópico vulvar.

Realiza un total de 15 sesiones con una energía acumulada de $4,959.9 \mathrm{~mJ} / \mathrm{cm}^{2}$.

Se constata mejoría del eritema y reducción de las lesiones extragenitales (Fig. 6). Actualmente presenta atrofia residual en la vulva, sin úlceras, por lo cual continúa en seguimiento y tratamiento con clobetasol ungüento al $0.05 \%$ dos veces a la semana.

\section{Discusión}

El liquen escleroso es un trastorno inflamatorio crónico, raro, que puede comprometer tanto el área genital como extragenital ${ }^{1,2}$.

Esta entidad fue descrita por primera vez por Hallopeau en 1887 como «liquen escleroso y atrófico» ${ }^{3}$. En los últimos años, se ha abandonado el calificativo "atrófico», ya que se reconoce que algunos casos están asociados con un epitelio hipertrófico en lugar de atrófico ${ }^{4}$.

Su prevalencia en la población general es de 1/3001,000 individuos y solo en un $15-20 \%$ compromete la

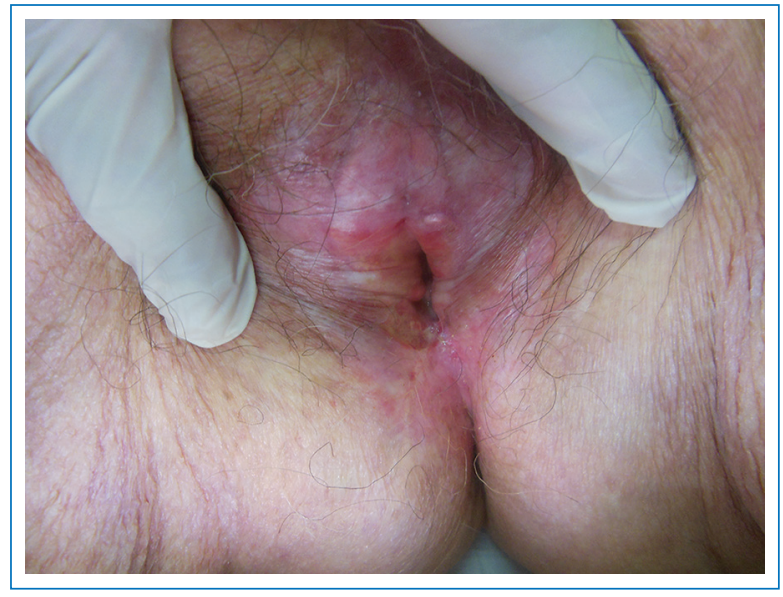

Figura 1. Vulva atrófica con úlcera con eritema perilesional y palidez blanquecina que se extiende a la región perineal y perianal en forma de número 8.

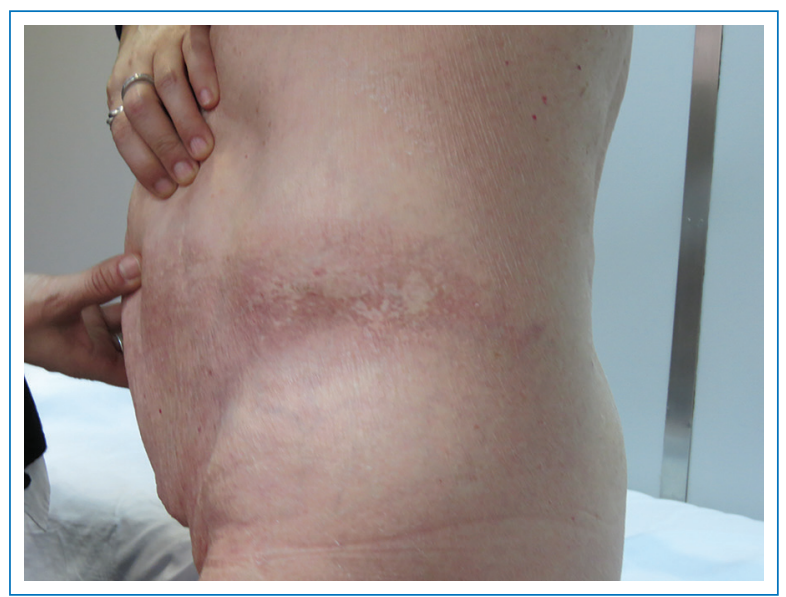

Figura 2. Placa atrófica en el flanco izquierdo, con coloración eritematoviolácea perilesional.

región extragenital ${ }^{2}$. Se presenta en todos los grupos de edad, pero aparece principalmente entre los $40 \mathrm{y}$ los 60 años ${ }^{5}$ y con mayor frecuencia en la mujer que en el hombre, con una relación de seis a uno ${ }^{3}$.

La etiología es desconocida. Se han involucrado factores: genéticos (HLA A29, B21, B40, B44 y Aw31), déficits enzimáticos (proteasas del tipo elastasas, 5-alfa-reductasa) hormonales (niveles bajos de testosterona), infecciosos (virus del papiloma humano [VPH], Borrelia burgdorferi, Mycobacterium fortuitum), traumáticos (se ha descrito el fenómeno isomórfico de Kôebner en cicatrices y después de traumatismos físicos, por fricción o tras quemaduras solares) e inmunológicos ${ }^{2,6}$. Se reporta que entre el 21.5 y el $34 \%$ de los pacientes con LE tienen 


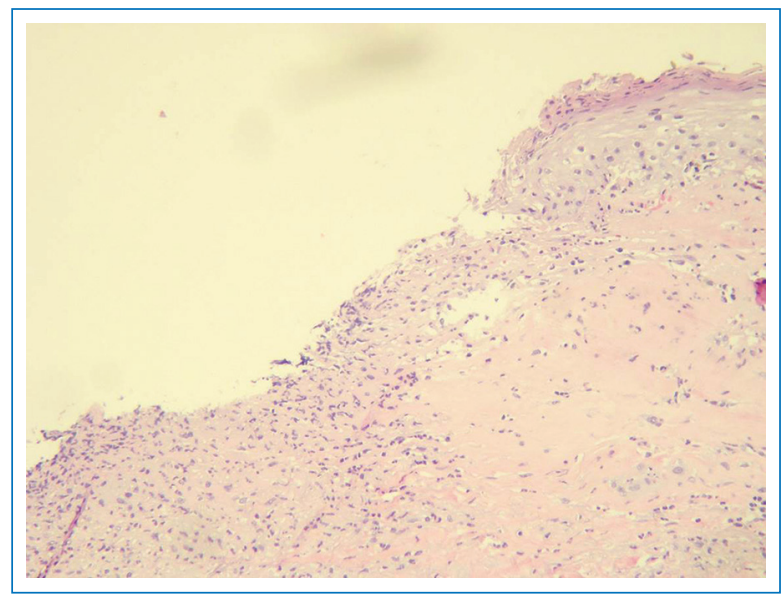

Figura 3. Histopatología vulva (H/E): presencia de úlcera y epidermis adelgazada con focos de vacuolización de la capa basal.

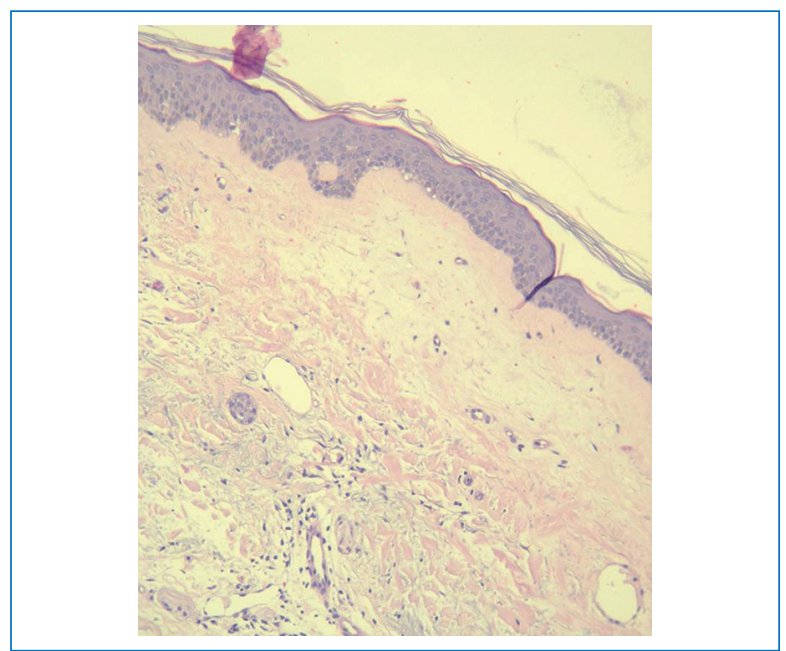

Figura 4. Histopatología flanco (H/E): epidermis adelgazada con focos de vacuolización de la capa basal, acompañada en la dermis de un infiltrado inflamatorio de tipo linfocitario y homogeneización del colágeno dérmico superficial.

un trastorno inmunitario asociado, como enfermedad tiroidea, alopecia areata, vitiligo y anemia perniciosa ${ }^{5}$.

Independientemente de la etiopatogenia, el resultado final es una disminución de las fibras elásticas y una destrucción de la matriz y las membranas basales ${ }^{6}$.

Clínicamente, a nivel vulvar comienza con eritema, que progresa a máculas y pápulas de color blanco marfil, brillantes, induradas y bien delimitadas, que tienden a confluir formando grandes placas. Afecta principalmente la parte interna de la vulva, el periné y

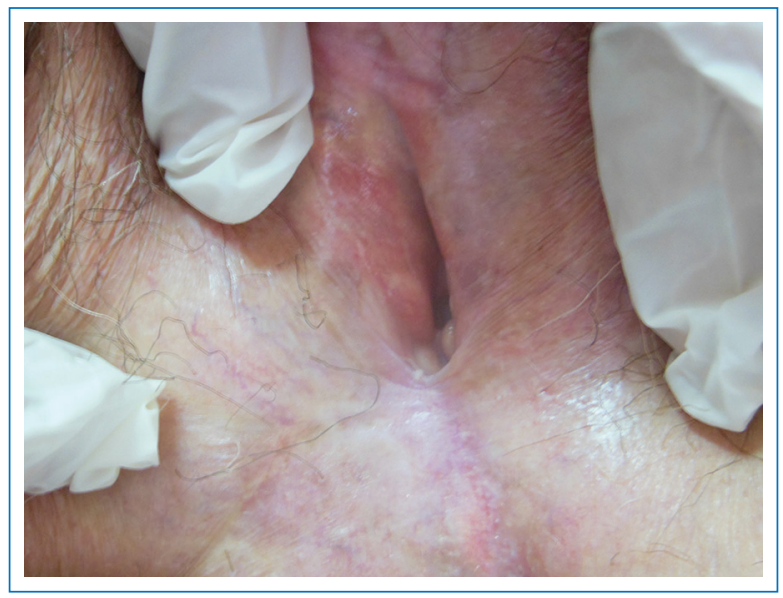

Figura 5. Vulva atrófica con resolución de la úlcera.

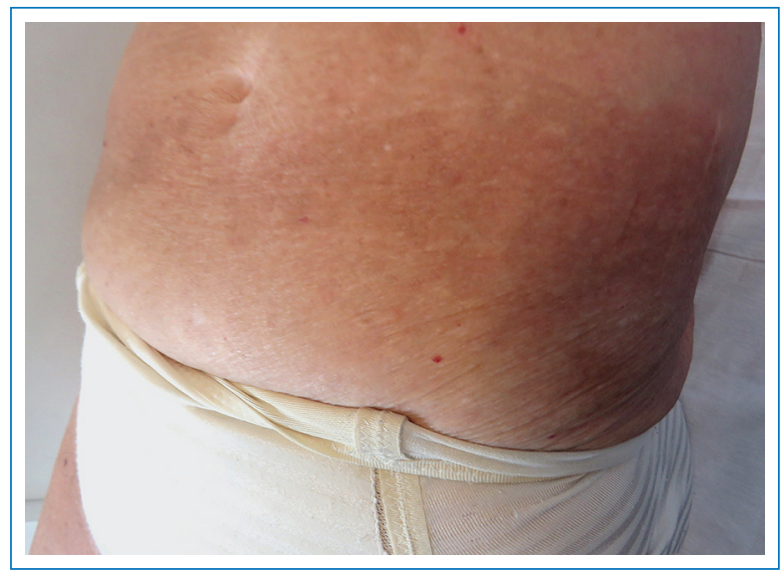

Figura 6. Mejoría clínica de placa en flanco izquierdo, con centro blanquecino atrófico y leve hiperpigmentación periférica.

el área perianal, con una forma típica en $\mathrm{ocho}^{5}$, como observamos en esta paciente.

Las lesiones de LE extragenital se localizan con mayor frecuencia en tronco, cuello, hombros y zonas de flexión, como muñeca y cadera ${ }^{7}$.

El síntoma predominante es el prurito a nivel vulvar y/o perianal, de intensidad variable, intermitente o permanente, que está presente en dos tercios de los casos $^{7}$. En cambio, las lesiones extragenitales son en su mayoría asintomáticas ${ }^{2}$.

Las complicaciones de LE descriptas en la mujer incluyen: desarrollo de carcinoma de células escamosas (3.5-5\%), formación de cicatrices (estrechamiento del introito, pseudoquiste del clítoris y estrechez del meato uretral), dolor neuropático (vulvodinia) y problemas psicosexuales 4 . 
El diagnóstico de liquen escleroso suele ser clínico. Cuando las características clínicas son típicas, el examen histológico no es esencial. Sin embargo, en las primeras etapas de la enfermedad, el diagnóstico puede ser difícil. Los principales diagnósticos diferenciales son liquen plano, liquen simple crónico, vitiligo, trastornos ampollares como el penfigoide de la membrana mucosa y la neoplasia intraepitelial vulvar ${ }^{8}$.

En nuestro caso, la clínica genital era sugestiva de liquen escleroso, pero se realizó biopsia de la misma con el fin de descartar posible evolución a carcinoma escamoso.

En cuanto a la histología, el LE presenta una epidermis disminuida, normal o aumentada o hiperqueratósica. Las uniones dermoepidérmicas se aplanan y debajo de ellas hay una banda de colágeno homogénea. Puede presentar, además, áreas focales de infiltrado de linfocitos, plasmocitos e histiocitos adyacentes a la unión dermoepidérmica ${ }^{6}$. En los casos clínicamente no concluyentes, el examen histológico es aconsejable, pero nunca debe interpretarse de forma aislada. Una histopatología inespecífica no descarta liquen escleroso, pero los hallazgos histológicos clásicos confirman el diagnóstico ${ }^{8}$.

Los objetivos del tratamiento incluyen el alivio de los síntomas, y la prevención de deformaciones anatómicas y de transformación maligna ${ }^{6}$.

El tratamiento de primera línea actual son los corticosteroides tópicos de alta potencia. La guía para el manejo del liquen escleroso, publicada por la British Association of Dermatologists en 2018, recomienda una terapia de inducción con propionato de clobetasol al $0.05 \%$ durante 3 meses (1 vez al día durante 1 mes, luego días alternos durante el mes siguiente y 2 veces a la semana en el mes restante $)^{4}$. En los meses 4 a 6 se propone 1 a 2 aplicaciones por semana o según necesidad 6 .

Los inhibidores tópicos de la calcineurina, como tacrolimus y pimecrolimus, son utilizados como terapia adyuvante, pero no han demostrado ser efectivos para su tratamiento inicial ${ }^{2}$.

En casos resistentes al tratamiento con corticosteroides 0 intolerancia, podemos recurrir a retinoides orales, metotrexato y fototerapia ${ }^{4}$.

Existen informes y pequeñas series de casos que han demostrado la efectividad de la terapia ultravioleta A (340-400 nm) y UVB-BA para el LE extragenital. Se ha demostrado que ambos aumentan los niveles de metaloproteinasa de matriz en piel humana y fibroblastos dérmicos cultivados, lo que puede explicar la efectividad en enfermedades de la piel esclerosante ${ }^{9}$.

Nuestra paciente mostró mejoría tanto clínica como sintomatológica de las lesiones genitales, pero no así de las lesiones extragenitales después de 6 meses de tratamiento combinado de clobetasol y tacrolimus, por lo que se indicó terapia con UVB-BA. La mayor disponibilidad y el costo favorable de UVB-BA en comparación con ultravioleta $A$, así como el menor efecto adverso de esta modalidad, lo convierten en una alternativa atractiva.

\section{Conclusiones}

La buena respuesta de nuestra paciente sugiere que la terapia con UVB-BA puede ser beneficiosa en el tratamiento del liquen escleroso extragenital, produciendo alivio sintomático y modificación del curso de la enfermedad.

\section{Conflicto de intereses}

Los autores declaran no tener ningún conflicto de intereses.

\section{Responsabilidades éticas}

Protección de personas y animales. Los autores declaran que para esta investigación no se han realizado experimentos en seres humanos ni en animales.

Confidencialidad de los datos. Los autores declaran que han seguido los protocolos de su centro de trabajo sobre la publicación de datos de pacientes.

Derecho a la privacidad y consentimiento informado. Los autores han obtenido el consentimiento informado de los pacientes y/o sujetos referidos en el artículo. Este documento obra en poder del autor de correspondencia.

\section{Bibliografía}

1. Vidarte-Orrego Gl. Liquen escleroso y atrófico extragenital en zona de presentación atípica. Dermatol Peru. 2018;28(3):164-6.

2. Zalazar ME, Bonetto VN, Salduna MD, Lascano AR, Kurpis M. Liquen Escleroso y Atrófico de localización extragenital. Rev argent dermatol;. 2018;99(3):31-40.

3. Serfaty JO, Castillón R, Cohen JP, Fumero DM, Reyes O. (2014). Liquen escleroso y atrófico reporte de un caso con revisión bibliográfica. Derm Ven. 2014;3:3-4.

4. Lewis FM, Tatnall FM, Velangi SS, Bunker CB, Kumar A, Brackenbury F et al. British Association of Dermatologists guidelines for the management of lichen sclerosus, 2018. Br J Dermatol. 2018;178(4):839-53.

5. Sánchez ABA, Vergara RFC. Liquen escleroso y atrófico vulvar. Dermatol Rev Mex. 2013; 57(5):394-7.

6. Baptista PV, Duarte S, Freitas D, Costa AR, Beires J. Líquen escleroso: revisão da literatura Lichen sclerosus: revision of the literature. Acta Obstet Ginecol Port. 2007;1(1):30-41.

7. Cabrera-Acea G, Hernández-Mompié J, Gómez-Cabrera C. (2016). Liquen escleroso y atrófico genital o craurosis de la vulva. Presentación de un caso. MediSur. 2016;14(6):796-800.

8. Fistarol SK, Itin PH. Diagnosis and treatment of lichen sclerosus. Am J Clin Dermatol. 2013;14(1):27-47.

9. Colbert RL, Chiang MP, Carlin CS, Fleming M. Progressive extragenital lichen sclerosus successfully treated with narrowband UV-B phototherapy. Arch Dermatol. 2007;143(1):19-20. 